\title{
Kilocalorie per Day
}

National Cancer Institute

\section{Source}

National Cancer Institute. Kilocalorie per Day. NCI Thesaurus. Code C139135.

A unit of energy equal to one kilocalorie per day. 